\title{
Clinical Analysis of Severe Pulmonary Fungal Infection in Intensive Care Unit
}

\author{
Qing Ye* \\ The People's Hospital of Tongcheng City, Tongcheng, Anhui 231400, China
}

\begin{abstract}
Objective: To analyze the characteristics of ICU patients with severe pulmonary fungal infection and the effect of preventive medicine. Method: The study group of 68 cases in the discovery of fungal infection associated with adverse symptoms before clinical implementation of preventive antibiotics, the control group of 52 cases in the discovery of symptoms and the diagnosis of fungal infection after symptomatic application of antibiotics. The efficacy of the two groups was compared and the characteristics of fungal infection were analyzed. Results: The effective rate of the study group was significantly higher than control group $(p<0.05)$. The proportion of Candida albicans was the highest, and was significantly higher than that of smooth Candida glabrata and Candida parapsilosis, $(p<0.01)$. Conclusion: For the treatment of ICU severe pulmonary fungal infection in patients with the use of prophylactic antibiotics can improve the treatment effect of fungal infection, clinical medication should pay attention to avoid abuse and analysis of the characteristics of drug use.
\end{abstract}

\author{
KEYWORDS \\ Intensive care unit \\ Pulmonary infection \\ Antibiotic application
}

\section{Introduction}

Pulmonary fungal infections are disease of bronchopulmonary caused by fungal, including primary and secondary pulmonary fungal infections. Fungal spores are inhaled into the lungs and the disease is called the primary pulmonary fungal infection. In other parts of the body, fungal infection is caused by the lymphatic or blood to the lungs, which is called secondary pulmonary fungal infection. Pulmonary fungal infection is very common in clinical setting, especially severe patients in ICU, because of the low immunity, large amount of antibiotics, intubation and other invasive treatment, therefore treatment of fungal infection is more difficult, but also increased the risk of the development of opportunity diseases in patients [1-2]. Prevention of fungal infection of patients with severe pulmonary infection in ICU, in order to reduce the degree of infection. In this paper, the characteristics and preventive

Copyright ( 2013 Qing Ye

doi: $10.18686 /$ aem.v2i1.1

Received: February 8, 2013; Accepted: April 17, 2013; Published online: June 4, 2013

This is an open-access article distributed under the terms of the Creative Commons Attribution Unported License (http://creativecommons.org/ licenses/by-nc/4.0/), which permits unrestricted use, distribution, and reproduction in any medium, provided the original work is properly cited.

${ }^{*}$ Corresponding author: The People's Hospital of Tongcheng City, Tongcheng, Anhui 231400, China. E-mail: yeyeq_722@126.com effect of patients in ICU with severe pulmonary fungal infection were analyzed.

\section{Materials and methods 2.1. Clinical data}

Selected patients in ICU of our hospital from January 2012 to December 2012 diagnosis by sputum culture confirmed severe pulmonary fungal infections in 120 cases as the research object. Approval by the hospital ethics committee and patient informed consent by clinicians, according to the diagnostic criteria of reference the 2011 American Thoracic Society in adult pulmonary and critical care patients with fungal infection treatment guidelines for the introduction [3]. According to the clinical application of antibiotics in patients, the study group was form by 68 cases, which 38 male and 30 female, and aged from 52 to 83 years old, with average $(22 \pm 5.3)$ years old. From there, 16 cases of severe craniocerebral injury, 5 cases of cerebral infarction, 8 cases of pulmonary infection, 15 cases of pulmonary edema, 2 cases of acute respiratory distress syndrome (66.3 cases). The control group was form with 52 cases, which 31 male and 21 female, and aged from $54-82$ years old, with the average $(65.8 \pm 4.8)$ years old. From a total 52 cases, there were 12 cases of severe brain injury, 4 cases of cerebral infarction, 15 cases of pulmonary infection, 6 cases of pulmonary edema, 13 cases of obstructive pulmonary emphysema, 2 cases of acute respiratory distress syndrome. 
There was no significant difference in gender, age and basic diseases between the two groups $(p>0.05)$.

\subsection{Method}

The study group was found to be associated with adverse symptoms of fungal infection in clinical implementation of preventive antibiotics, the control group was found in symptoms and diagnosis of fungal infection after symptomatic application of antibiotics. Including types of antibiotics: fluconazole, amphotericin B and voriconazole. At the same time according to the test results of sputum fungal infection characteristics.

\subsection{Efficacy evaluation criteria}

Fungal infection in patients with therapeutic effect by clinical symptoms improvement (asthma, cough, expectoration) and signs improve (pulmonary rales, temperature changes), image results (lung shadow disappeared) comprehensive evaluation. The results divided into recovery, improvement, and invalid, while the treatment efficiency = (cure and improvement)/number of research cases $\times 100 \%$.

\subsection{Statistical processing}

Data were processed and analyzed by software SPSS 18.0, and data were analyzed by chi square test. When $p<0.05$, the difference was statistically significant.

\section{Results}

\subsection{Comparison of curative effect}

Clinical efficacy in two groups of patients in ICU with severe pulmonary fungal infection, wherein study group was significantly higher than control group, and the difference was statistically significant $(p<0.05)$. From there, 1 patients from the study group were significantly worse; whereas for control group, there were 6 patients treated with invalid, and 4 cases from there were worsen and 2 cases were died.

Table 1. Comparison of treatment effect of two groups of patients with pulmonary fungal infection (cases \%).

\begin{tabular}{cccccc}
\hline Group & Cases & Cure & Improving & Invalid & $\begin{array}{c}\text { Treatment } \\
\text { efficiency (\%) }\end{array}$ \\
\hline Research group & 68 & 30 & 37 & 1 & 98.52 \\
Control group & 52 & 20 & 26 & 6 & 88.46 \\
$x^{2}$ & - & - & - & - & 5.437 \\
$p$ & - & - & - & - & $<0.05$ \\
\hline
\end{tabular}

\subsection{Fungal infection characteristics}

The results of sputum culture in 120 cases of severe pulmonary fungal infection with patients in ICU, as shown in Table 2, the proportion of Candida albicans was the highest, which was significantly higher than Candida glabrata and Candida parapsilosis, and the difference between data was statistically significant $\left(x^{2}=147.635,161.186, p<0.01\right)$.
Table 2. 120 cases of fungal infection in patients with pulmonary fungal infection.

\begin{tabular}{ccc}
\hline Fungal type & Cases & Proportion (\%) \\
\hline Candida albicans & 104 & 86.67 \\
Candida glabrata & 10 & 8.33 \\
Candida parapsolosis & 6 & 5.00 \\
Total & 120 & 100 \\
\hline
\end{tabular}

\subsection{Risk factors analysis}

In this study, more than 2 kinds long-term use of corticosteroids and immunosuppressive agents in patients were 72 cases, which accounted for $60 \%$ from the total number. Among them, 26 patients had long-term application of glucocorticoids, which accounted for $21.67 \%$, and 33 patients had long-term application of immunosuppressive agents, accounted for $27.50 \%$. Besides that, 45 patients with low serum protein, accounted for $37.50 \%, 32$ cases of low lymphocytes, accounted for $26.67 \%$ cases, and patients with tracheostomy was 28 cases, accounted for $31.67 \%$ cases. Moreover, 38 patients that hospitalize more than one week, accounted for $31.67 \%$, and there were 80 cases of patients that using two or more broad-spectrum antifungal drug which hospitalized more than 1 week, accounted for $66.67 \%$. Specific results are shown in Table 3.

Table 3. 120 risk factors of pulmonary fungal infection in patients.

\begin{tabular}{ccc}
\hline Risk factors & Cases & Proportion (\%) \\
\hline $\begin{array}{c}\text { Long term application of glucocorticoids } \\
\text { Long term application of }\end{array}$ & 26 & 21.67 \\
immunosuppressive agents & 33 & 27.50 \\
Low serum protein & 45 & 37.50 \\
Low lymphocytes & 32 & 26.67 \\
Hospitalized more than 1 weeks & 38 & 31.67 \\
Tracheal intubation and tracheotomy & 28 & 31.67 \\
$\begin{array}{c}\text { Using two or more broad-spectrum and } \\
\text { highly effective drug, hospitalized more } \\
\text { than 1 weeks }\end{array}$ & 80 & 66.67 \\
Two factors above & 72 & 60.00 \\
\hline
\end{tabular}

\section{Discussion}

ICU patients with serious illness, which their body's immune function defects, and easy encounter with lung infection that trigger by various factors, especially in most patients with invasive treatment, such as catheter placement in vivo, which provides favorable conditions for fungal infection [4]. The clinical symptoms and signs are not specific to pulmonary fungal infection, such as cough, expectoration, fever, psychiatric symptom, which is similar with most basic diseases and common bacterial infection. Hence it not easy to discover on time, and delayed the diagnosis, which greatly affected the effectiveness of the medication and treatment [5-6]. Pulmonary fungal infec- 
tion further reduce patients' immunity, causing tissue injury, and even worsen the primary diseases, induce all sorts of complication; therefore, timely treatment to improve the patients symptoms and improve the quality of life was significance important.

In this study, 1 patients from the study group were deteriorate, and 6 patients from control group were treated with invalid, from there 2 patients died of heart failure, and 4 patients had worse condition, therefore effective rate of study group was significantly higher than that of control group, and the difference was statistically significant $(p<$ 0.05 ). It is not difficult to see that implementation of preventive antibiotics in study group can effectively reduce the clinical symptoms, has a good clinical application effect. It is not difficult to see that the proportion of Candida albicans was the highest in this study. The highest proportion of Candida albicans in Table 2 was significantly higher than that of Candida glabrata and Candida parapsilosis. The difference between the data was statistically significant $\left(x^{2}=\right.$ $147.635,161.186, p<0.01)$. Therefore, in the prophylactic use of drug, it should pay attention to the corresponding symptoms caused by different microbes, to increase the effectiveness and efficiency of the treatment.

Prophylactic drugs purpose was used before unknown clinical diagnosis of patients with infection, and the application of antibiotics for anti-inflammatory treatment. Once the patient carry a pathogenic fungus, drugs can quickly play a role in the treatment, to achieve the purpose of early treatment and recovery. In this group of patients, the use of prophylactic medicine in clinical treatment which make the effective rate significantly higher than control group of patients with fungal infection, suggesting that the clinical application of prophylactic antibiotics is beneficial to improve the treatment of ICU patients with severe pulmonary fungal infection. It not only reduce the patient's pain, but also conducive to the prevention of early complications and primary diseases. But it is worth to pointing out that the abuse of antibiotics has become the most discussed topic, hence the application of sputum culture confirmed the infection was caused by relative microbes and the application of symptomatic medication will more conducive and avoid the misuse of drugs. Because preventive medicine is beneficial to improve the treatment effect of pulmonary fungal infection, hence it should further investigation of the characteristics of pulmonary fungal infection, so as to facilitate the usage of prophylactic drugs, to avoid drug abuse. In addition should avoid the long-term use of antibiotic, to prevent the develop of drug's resistant.

Candida albicans, Candida glabrata and Candida parapsilosiswere are the main pathogenic microbes that induce severe pulmonary fungal infection in ICU. Fluconazole is a broad-spectrum antifungal drug that use for the treatment of infection It has a good inhibitory effect of common fungal infection and better effect in term of oral drug absorption. For fasting oral medication, the absorption rate achieving $90 \%$ and not easy to be affected by diet, $\mathrm{H}_{2}$ receptor blockers and anti-acid drugs. It subjected to renal excretion and having minimum side effect. Amphotericin $\mathrm{B}$ is a polyene antifungal medication, but the side effects of the drug are relatively large, which the administration prone to the adverse reactions such as nausea and vomiting, chills, headache, anemia, and white blood cells decreased. Administration of the drug should be cautious where medication attention to patients with signs of change and timely evaluate the effect.

In conclusion, the treatment effect of prophylactic antimicrobials drugs for patients in ICU with severe pulmonary fungal infection can improve the therapeutic effect of antifungal, and accelerate the speed of disease remission. Candida albicans causing the major infection and the clinical use of the antimicrobials drugs should be rational to prevent the drug abuse.

\section{References}

1. Shao HZ. Comparison and analysis of X-ray and CT diagnosis of pulmonary fungal infection. Chinese Journal of Practical Medicine. 2013;40(2):52-54.

2. Liu JL, Lv XD, Zhang YQ. 70 years of age or older patients with chronic obstructive pulmonary disease with pulmonary fungal infection, diagnosis and analysis. International Respiratory Journal. 2012;32(21):1616-1618.

3. Han PW, Zhang H, et al. 2011 American Thoracic Society in adult pulmonary and critical care patients with fungal infection treatment guidelines profile. International Journal of Respiration. 2011;31(8):561-562.

4. Wang GH. Clinical analysis of 72 cases of pulmonary fungal infection. Chinese Modern Pharmaceutical Applications. 2013;7(2):18-20.

5. Jiang N, Huang J. Clinical analysis of nosocomial pulmonary fungal infection in patients in Department of Respiration. Chinese Medicine Guide. 2013;11(1):211-212.

6. Liu ZY. The clinical characteristics of pulmonary fungal infection in elderly patients. Chinese Medicine Guide. 2012;20(10):63-65. 